Tropical Journal of Pharmaceutical Research April 2017; 16 (4): 837-847

ISSN: $1596-5996$ (print); 1596-9827 (electronic)

(C) Pharmacotherapy Group, Faculty of Pharmacy, University of Benin, Benin City, 300001 Nigeria.

All rights reserved.

Available online at http://www.tjpr.org

Original Research Article

http://dx.doi.org/10.4314/tjpr.v16i4.13

\title{
Assessment of absorption of four lignan constituents of JingNing particles in rat gut using in situ single-pass intestinal perfusion
}

\author{
Chunjing Yang ${ }^{1}$, XingBin Yin ${ }^{1}$, Xiaoxv Dong ${ }^{1}$, Jing $\mathrm{Fu}^{1}$, Wenping Wang ${ }^{1}$, \\ Xueying Du${ }^{1}$, Long tai You ${ }^{1}$, Lin Guo ${ }^{1}$, Sali $\mathrm{Cao}^{1}$, Huyiligeqi $i^{1,2}$ and Jian $\mathrm{Ni}^{1 *}$ \\ ${ }^{1}$ School of Chinese Materia Medica, Beijing University of Chinese Medicine, Beijing 100102, ${ }^{2}$ Affiliated Hospital, Inner Mongolia \\ University for Nationalities, Tongliao 028000, China
}

*For correspondence: Email: njtcm@263.net; Tel: 010-84738607

Received: 16 November 2016

Revised accepted: 15 March 2017

\begin{abstract}
Purpose: To study small intestinal absorption of schisadrol $A$, schisandrol $B$, schizandrin $A$ and schisandrin B in JingNing particles using in situ single-pass intestinal perfusion (SPIP).

Methods: Absorption rate constant (Ka) and apparent permeability (Papp) of the drugs at different concentrations in various parts of rat small intestine (duodenum, jejunum and ileum) were determined using SPIP. JingNing particles were also perfused in situ at different $\mathrm{pH}$ in the entire rat intestine. Ethanol extract of Schisandra chinensis (standard) at low concentration was perfused in the duodenum for comparison with extract of JingNing particles.

Results: The order of apparent permeability of the four lignans was schisandrol $A<$ schisandrol $B<$ schizandrin $A<$ schisandrin B. Ka and Papp values of the four lignans in JingNing particles were concentration-dependent. Absorption increased in the rank order: ileum > duodenum > jejunum. Optimum absorption $\mathrm{pH}$ was 6.50. Polygala tenuifolia extract and volatile oil of Rhizoma acori tatarinowii significantly $(p<0.05)$ enhanced the absorption of the four lignans.

Conclusion: The four lignans were well absorbed in the intestinal tract, particularly the ileum, probably through carrier-mediated transport. The alcohol extract of Polygala tenuifolia and volatile oil of Rhizoma acori graminei enhanced the absorption of the four lignans.
\end{abstract}

Keywords: JingNing, Intestinal absorption, Polygala tenuifolia, Rhizoma acori graminei, Lignans, Schisandrol, Schisandrin, Single-pass intestinal perfusion

Tropical Journal of Pharmaceutical Research is indexed by Science Citation Index (SciSearch), Scopus, International Pharmaceutical Abstract, Chemical Abstracts, Embase, Index Copernicus, EBSCO, African Index Medicus, JournalSeek, Journal Citation Reports/Science Edition, Directory of Open Access Journals (DOAJ), African Journal Online, Bioline International, Open-J-Gate and Pharmacy Abstracts

\section{INTRODUCTION}

JingNing particles, containing Radix Pseudostellariae, Radix Rehmanniae praeparata, Schisandra chinensis (Turcz.)Baill, Polygala tenuifolia, Rhizoma acori tatarinowii, Poria cocos (Schw.), Wolf and Barbary wolfberry fruit, is a traditional Chinese medical formula with a long history of its use in clinical practice for treating attention deficit hyperactivity (ADHD). The alcohol extract of Schisandra chinensis fruit is one of the components of traditional Chinese herbal medicines in the JingNing particles used as hepato-protective agents $[1,2]$. Schisandra chinensis fruit has been used for thousands of years in the management of insomnia, coronary heart disease, skin disorders, depression and menopausal symptoms $[3,4]$.

The lignans of Schisandra chinensis fruit can enhance concentration and mental performance. They are used as adjuvant substances in the 
treatment of ADHD, Alzheimer's disease, Parkinsonism, and Meniere's diseases [5]. Traditional theories believe that endogenous liver wind is associated with ADHA.

Food and Drug Administration (FDA) has recognized that the poor drug permeation across the intestinal mucosa is one of the common causes for drugs absorption failure and low drug bioavailability $[6,7]$. The factors of influencing drug absorption contain the physical and chemical properties of compounds, such as structure, pKa, dosage forms characteristics, etc [8]. The majority of drugs are administered through the oral route, especially for traditional Chinese medicine [9]. The small intestine is the main absorption site of drugs. There are some methods used to evaluate intestinal absorption kinetics. These include in vivo, in vitro and in situ methods [10-12]. In situ methods have many advantages in that they provide viable intestinal mucosa, nerve system and blood flow, as well as the expression of specific enzymes and transporters [13]. Single-pass intestinal perfusion is widely used for studying intestinal absorption of drugs due to its high degree of accuracy [1417]. This study was aimed at investigating the intestinal absorption of four lignans, the main and effective ingredients in the Schisandra chinensis (a major constituents of JingNing particles) using SPIP.

\section{EXPERIMENTAL}

\section{Materials}

Schisandrol A, schisandrol B, schizandrin A and schisandrin B (purity $\geq 98 \%$ ) were bought from the National Institutes for Food and Drug Control, Beijing, China. JingNing particles were made in the laboratory as follows: Schisandra chinensis and polygala tenuifolia were extracted with $60 \%$ ethanol; essential oils were extracted from Rhizomaacori graminei; while the other drugs were extracted with water. The culture and perfusion solution was Krebs-Ringer (K-R) culture solution. Acetonitrile, methanol were purchased from Sigma. Double-distilled water was used during the entire HPLC procedure. All other chemical materials were of analytical grade and bought from commercial suppliers.

\section{Animals}

Male Sprague Dawley (SD) rats (mean weight, $250 \pm 20 \mathrm{~g}$ ) were obtained from the Weitonglihua Lab Animal Services Center (Changping, Beijing, China). The rats were fasted overnight but allowed free access to clean drinking water before starting the experiment. The experimental procedures were in compliance with the National Institutes of Health Guide for Care and Use of Laboratory Animals [18] and were approved by the Committee on Animal Care and Usage of the Beijing University of Chinese Medicine (approval no. BUCM-2-2017010101-1001).

\section{Preparation of drug solutions}

Sodium dodecyl sulfate (SDS, $0.2 \%$ ) was added to K-R solution to help dissolve JingNing particles. The $\mathrm{pH}$ of $\mathrm{K}-\mathrm{R}$ solution was adjusted to 6.5 with $\mathrm{HCl}$ and $\mathrm{NaOH}$.

\section{Determination of Schisadrol A, Schisandrol B, Schizandrin A and Schisandrin B by HPLC}

The HPLC system involved a LC-20AT (Japan), fitted with an aglient-C18 $(250 \times 4.6 \mathrm{~mm}, 5 \mu \mathrm{m})$ column. The mobile phase were acetonitrilewater. The detection wavelength for Schisadrol A, Schisandrol B, Schizandrin A and Schisandrin $B$ was $215 \mathrm{~nm}$. The specificity was described by comparing the chromatographs of several different types of solutions, including a drug-free $\mathrm{K}-\mathrm{R}$ solution, the intestinal perfusion solution of JingNing particles and the reference solution of schisandrol $A$, schisandrol $B$, schizandrin $A$ and schisandrin B [19]. The linearity was established by standards sample. The sample was diluted into different concentrations [20]. The linearity range of this method was $0.40-50.20 \mu \mathrm{g} / \mathrm{ml}$. Recovery was expressed as in percentage terms. Precision was expressed as relative standard deviation (RSD). Intra-day precision was measured during one day by analyzing six replicates of intestinal perfusion samples, while inter-day precision was detected on five consecutive days for the intestinal perfusion samples. Stability was tested within $24 \mathrm{~h}$.

\section{Studies on drug stability and adsorption}

A drug-free intestinal circulation fluid was made from a K-R culture solution and perfused in rat intestine for $3 \mathrm{~h}$. JingNing particles were dissolved in the blank intestinal circulation fluid and incubated at $37{ }^{\circ} \mathrm{C}$. Aliquots of $1 \mathrm{~mL}$ samples were taken out from the intestinal tract at different time intervals $(0,1,2$ and $3 \mathrm{~h})$ for analysis.

\section{Perfusion experiment}

The perfusion experiment was conducted as previously described [14,19]. Briefly, Male SD rats were used in situ SPIP studies. Open abdomen of the rat along the midline. The jejunum was cut from approximately $15 \mathrm{~cm}$ below the pylorus, and the ileum was cut from 20 
$\mathrm{cm}$ above the caecum. For each section, $10 \mathrm{~cm}^{3}$ segments were cut. Medicated K-R of a known weight was infused into the intestine at a high flow rate until the intestine was filled up. Then reduced the flow rate for a while. At steady state, collect intestinal perfusate samples at different time.

Samples from perfusion studies were diluted with ethanol $(1: 1, \mathrm{v}: \mathrm{v})$ and centrifuged for $20 \mathrm{~min}$ at $13000 \mathrm{r} / \mathrm{min}$. The resultant supernatants were injected into the HPLC column for analyses.

\section{Data treatment}

Eqs 1 and 2 were used to process the data [20,21]:

$\mathrm{Ka}=\left(1-\frac{\text { pout }}{\text { pin }} \cdot \frac{\text { vout }}{\text { Fin }_{n}}\right) \cdot \frac{v}{\pi \mathrm{i}^{2}}$

Popp $=\frac{-v \cdot \ln \left(\frac{p o u t}{p \text { in }} \frac{v o u r}{V i n}\right)}{2 \pi \% l}$.

where $V_{\text {in }}$ and $V_{\text {out }}$ are the respective inlet and collected outlet volumes at steady state (The gravimetric correction method proposed by Sutton et al was used; $v$ is the flow rate $(0.20$ $\mathrm{ml} / \mathrm{min}) . \rho_{\text {in }}$ and $\rho_{\text {out }}$ are the respective inlet and collected outlet concentration at steady state $(\mu \mathrm{g} / \mathrm{ml})$, and $l$ and $\gamma$ are the length $(\mathrm{cm})$ and radius $(\mathrm{cm})$ of the mouse intestine, respectively.

\section{Statistics}

All values are expressed as mean \pm standard deviation (SD). SPSS version of 17.0 software was used for statistical analysis, and $p<0.05$ was considered statistically significant [22].

\section{RESULTS}

\section{Validation of the HPLC method}

The mobile phase was shown in Table 1. Schisandrol $A$, schisandrol $B$, schizandrin $A$ and schisandrin $B$ exhibited major well-resolved peaks at approximately $14.5,17.5,33.5,38.5$ min respectively, in HPLC as shown in Figure 1. The calibration curve was linear in the range of $0.40-50.15 \mu \mathrm{g} / \mathrm{ml}$ of schisandrol A, 0.40 $50.10 \mu \mathrm{g} / \mathrm{ml}$ of schisandrol B, $0.42-52.95 \mu \mathrm{g} / \mathrm{ml}$ of schizandrin A and $0.40-50.20 \mu \mathrm{g} / \mathrm{ml}$ of schizandrin B.

The stability and precision of the analytical method were within statistical limits $(\mathrm{RSD}<3 \%$, $n=6$ ). These results showed that the HPLC method established was suitable [22] (Table 2).
Table 1: Mobile phase of gradient elution

\begin{tabular}{lcc}
\hline $\begin{array}{l}\text { Time } \\
(\boldsymbol{m i n})\end{array}$ & Acetonitrile (\%) & $\begin{array}{c}\text { Water } \\
(\%)\end{array}$ \\
\hline 0 & 40 & 60 \\
20 & 65 & 35 \\
45 & 75 & 25 \\
47 & 40 & 60 \\
57 & 40 & 60 \\
\hline
\end{tabular}

\section{Studies on the drug stability and adsorption}

The results (Table 2) indicated that the stability of the four chemical compounds in blank intestinal circulation fluid had RSD $<2 \%(n=6)$. They were deemed to be stable in K-R intestinal circulation fluid when intestine was perfused at $37{ }^{\circ} \mathrm{C}$ for $3 \mathrm{~h}$.

\section{Concentration-dependence of absorption profiles of the four lignans in JingNing particles}

The absorption profiles of the four lignans in JingNing particles at different concentrations $(6.4,12.8$ and $25.6 \mathrm{mg} / \mathrm{ml})$ in the duodenum, jejunum and ileum were shown in Figure 2 and Figure 3. There was a non-linear increase corresponding to increases in the concentrations of JingNing particles in the intestinal circulation fluid. However, the absorptions of the four lignans of JingNing particles decreased as their concentrations increased. The results showed that the transport and absorption of schisandrol A, schisandrol B, schizandrin A and Schisandrin $B$ would be saturated at high concentrations

\section{Absorptive profiles of the four lignans}

$\mathrm{Ka}$ and Papp values in the presence of JingNing particles in the different intestinal segments were shown in Figure 4 and Figure 5. lleum produced the best results $(p<0.05)$, followed by the jejunum, and the duodenum, in that order. However, there were no significant differences between the jejunum and the duodenum $(p<$ $0.05)$. The four lignans were well absorbed in the whole intestine.

\section{Influence of other components of JingNing on absorption profiles of the four components}

The alcohol extract of Schisandra chinensis at a concentration of $2 \mathrm{mg} / \mathrm{ml}$ was chosen as the standard. The segment of intestine used was the jejunum. The results showed that Polygala tenuifolia significantly enhanced the absorption of schizandrol A (200\%), schizandrol B (250\%), Schisandrin A (380\%) and schisandrin B (280 
$\%)$ in the jejunum. The volatile oil of Rhizoma acori tatarinowii enhanced the absorption of schizandrol A (300\%), schizandrol B (190\%), schisandrin A (340\%) and schisandrin B (240
$\%)$, relative to the standard. However, the other components of JingNing had no obvious effects on the absorption of the four lignans (Figure 7).

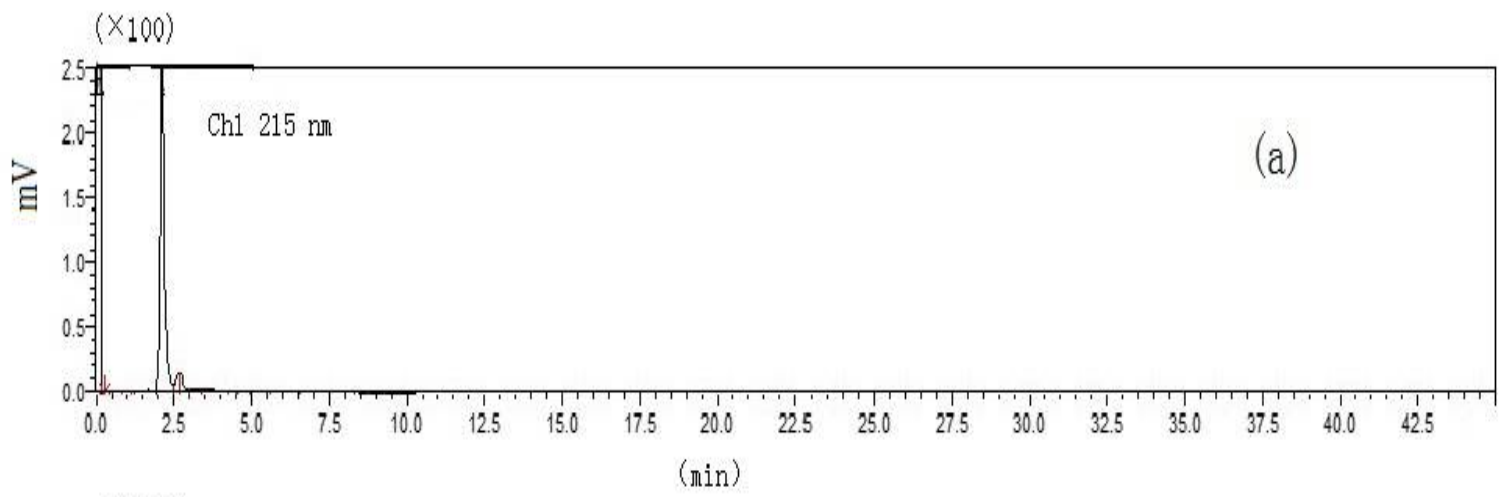

$(\times 100)$

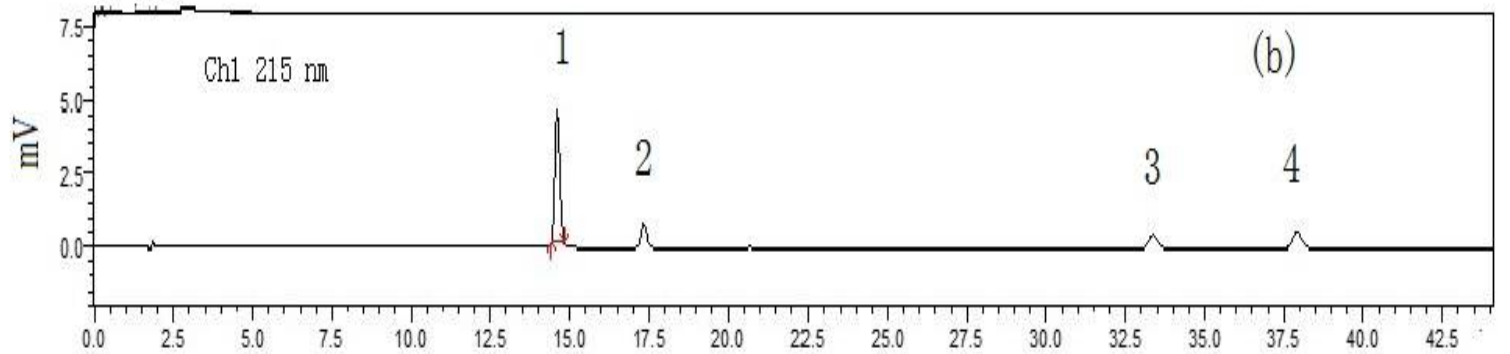

$(\min )$

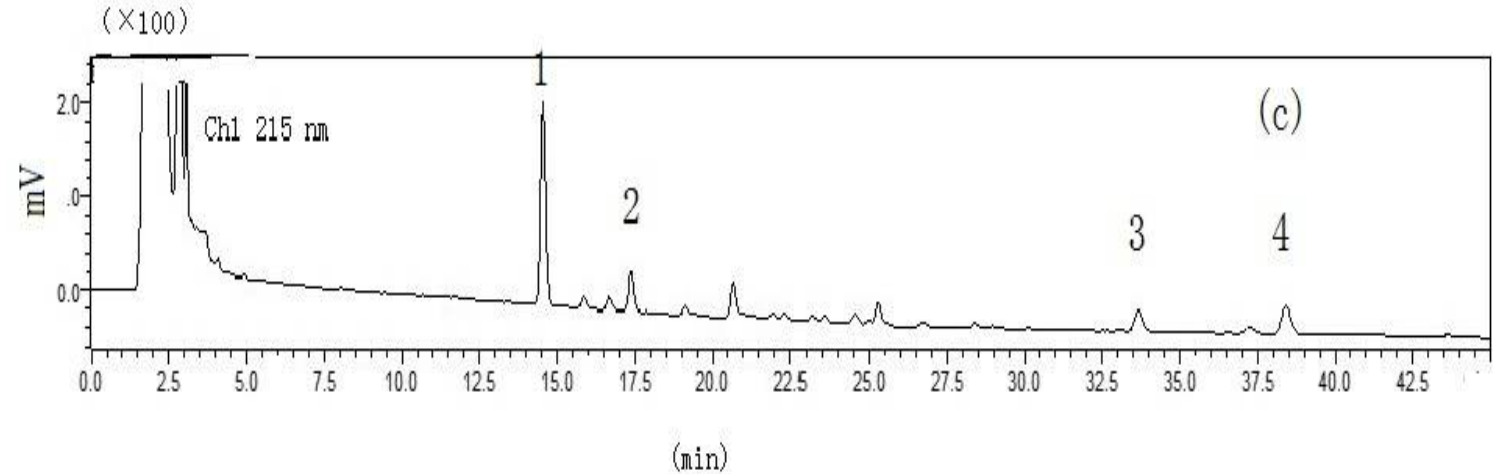

Figure 1: Specificity results. K-R solution (a), standard solution (b), sample solution of JingNing particles (c). schisandrol A (1), schisandrol B (2), schizandrin A (3), schizandrin B (4)

Table 2: Accuracy, stability and precision of analysis of the four lignans in serosal fluid

\begin{tabular}{lcccc}
\hline $\begin{array}{l}\text { Concentration } \\
\text { level }\end{array}$ & Sample & Accuracy & Stability (0-24h) & Precision (RSD) \\
\hline \multirow{2}{*}{ High } & Schisandrol A & 1.02 & $0.40 \%$ & $0.61 \%$ \\
concentration & Schisandrol B & 0.99 & $0.40 \%$ & $1.04 \%$ \\
& Schizandrin A & 0.95 & $0.65 \%$ & $0.43 \%$ \\
Middle & Schizandrin B & 0.95 & $0.40 \%$ & $0.25 \%$ \\
concentration & Schisandrol A & 1.03 & $0.38 \%$ & $0.44 \%$ \\
& Schisandrol B & 1.01 & $0.38 \%$ & $1.26 \%$ \\
& Schizandrin A & 0.99 & $0.38 \%$ & $0.57 \%$ \\
Low & Schizandrin B & 0.97 & $0.39 \%$ & $0.35 \%$ \\
concentration & Schisandrol A & 1.01 & $0.29 \%$ & $0.83 \%$ \\
& Schisandrol B & 1.01 & $0.29 \%$ & $0.95 \%$ \\
& Schizandrin A & 0.96 & $0.33 \%$ & $1.27 \%$ \\
\hline
\end{tabular}



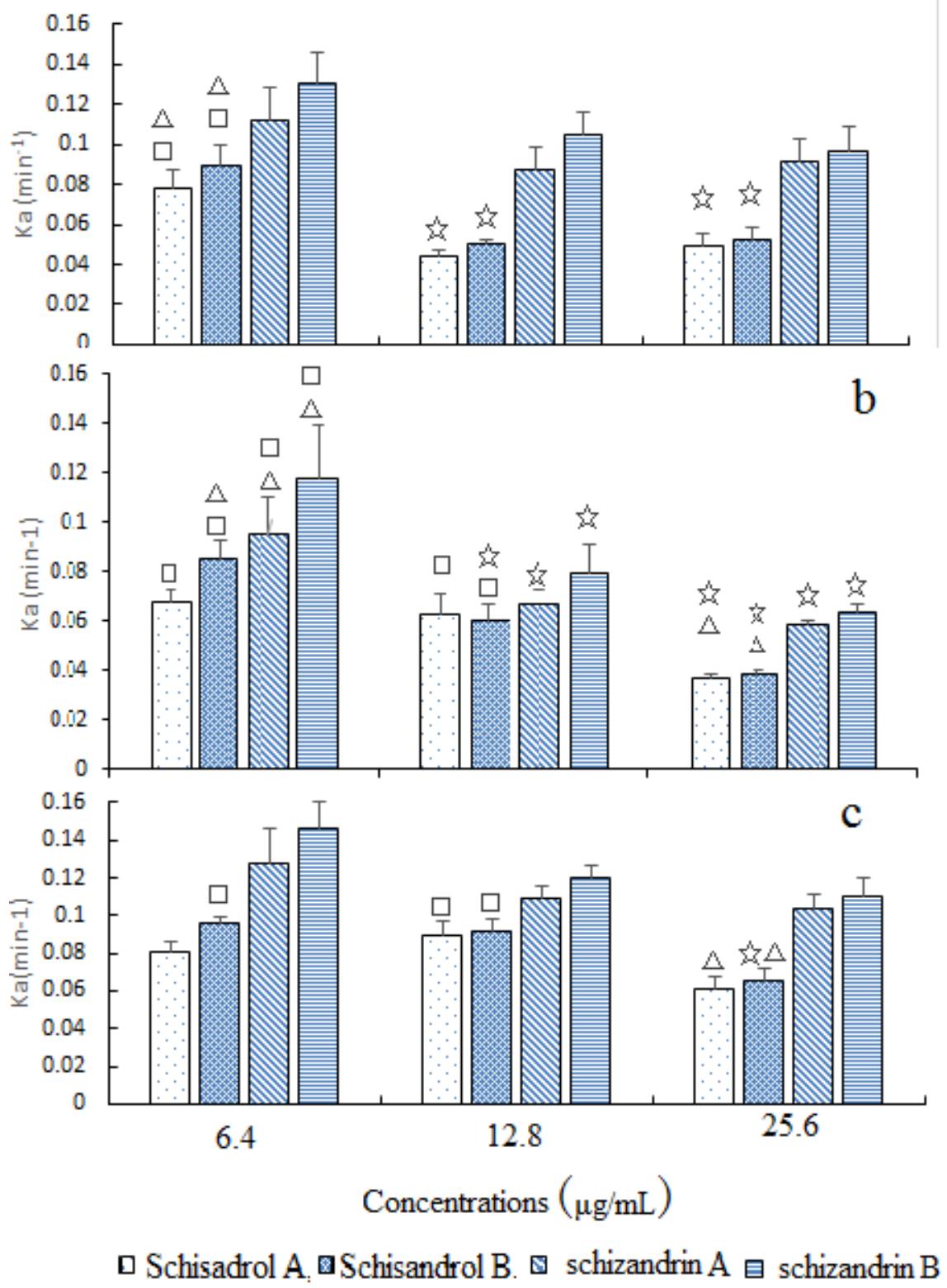

Figure 2: The absorption rate constant (Ka) of the four lignans in duodenum (a), jejunum (b), ileum (c); is $p<$ 0.05 , VS Ka of low concentration; $\Delta p<0.05$, VS. Ka of middle concentration; $\square p<0.05$, VS. Ka of high concentration

\section{DISCUSSION}

The purpose of the research was to determine the absorption site of the four lignans in extract of JingNing particles as well as the $\mathrm{pH}$ for their optimum absorption, and the influence of herbherb interaction on their intestinal absorption and bioavailability.

The results of concentration dependence showed a non-linear increase in the transport and absorption with different concentrations. With increase in concentration, absorption attained saturation, indicating that the absorption of the four lignans in JingNing particles may be carrier- mediated. The $\mathrm{pH}$ range of the intestinal tract is 5 - 7. The $\mathrm{pH}$ value of intestinal sites decreases in the order of ileum $>$ jejunum $>$ duodenum. Thus the $\mathrm{pH}$ of the ileum is close to 7 , which corresponds to the optimum $\mathrm{pH}$ for the absorption of the four lignans in JingNing particles. The lignanoids in Schisandra chinensis are the substrates for Pglycoprotein (P-gp), whose expression in the duodenum, jejunum, and ileum was increased. The increase may account for the duodenum being the best absorption site for Fructus schisandrae. However, the ileum was the best absorption site for the total lignanoids in JingNing particles. The possible reason for this might be that some components in Polygala tenuifolia and 
Rhizoma acori tatarinowii might compete with Fructus schisandrae for binding to P-gp.

Studies have shown that absorption is slow when Papp $<1.8 \times 10^{-4} \mathrm{~cm} / \mathrm{min}$, but enhanced when
Papp $>1.2 \times 10^{-3} \mathrm{~cm} / \mathrm{min}$ [23]. The results obtained in this study showed that Papp values of the four lignans were higher than $1.2 \times 10^{-3}$ $\mathrm{cm} / \mathrm{min}$, suggesting that they were well absorbed in the intestine.

a
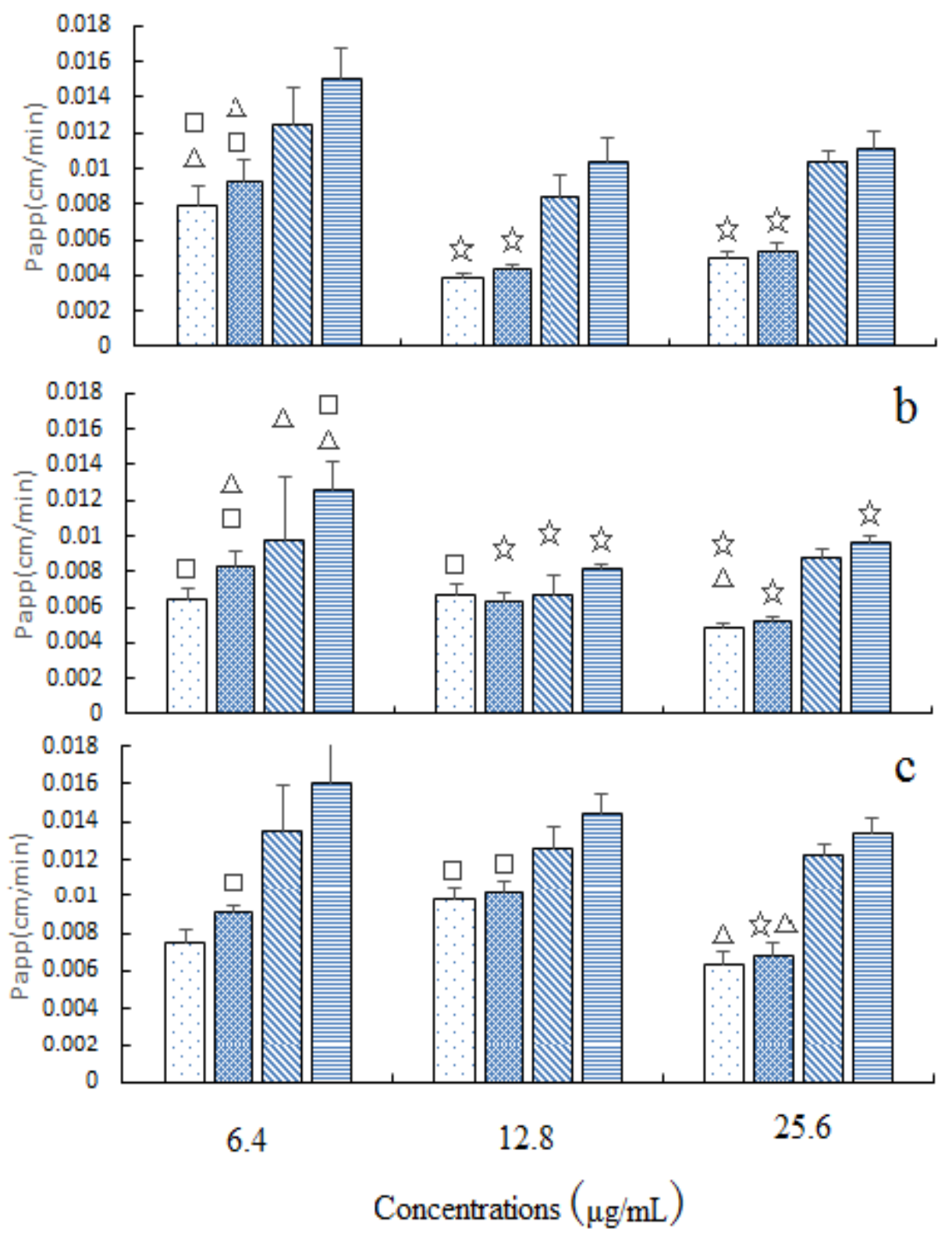

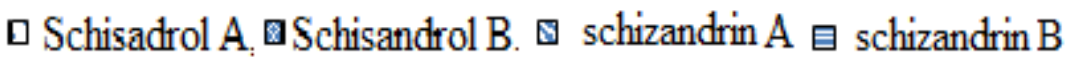

Figure 3: Apparent permeability (Papp) of the four lignans in duodenum (a), jejunum (b), ileum (c); is $p<0.05$, VS. Papp of the low concentration; $\Delta p<0.05$, VS. Papp of middle concentration; $\square p<0.05$, VS. Papp of high concentration) 

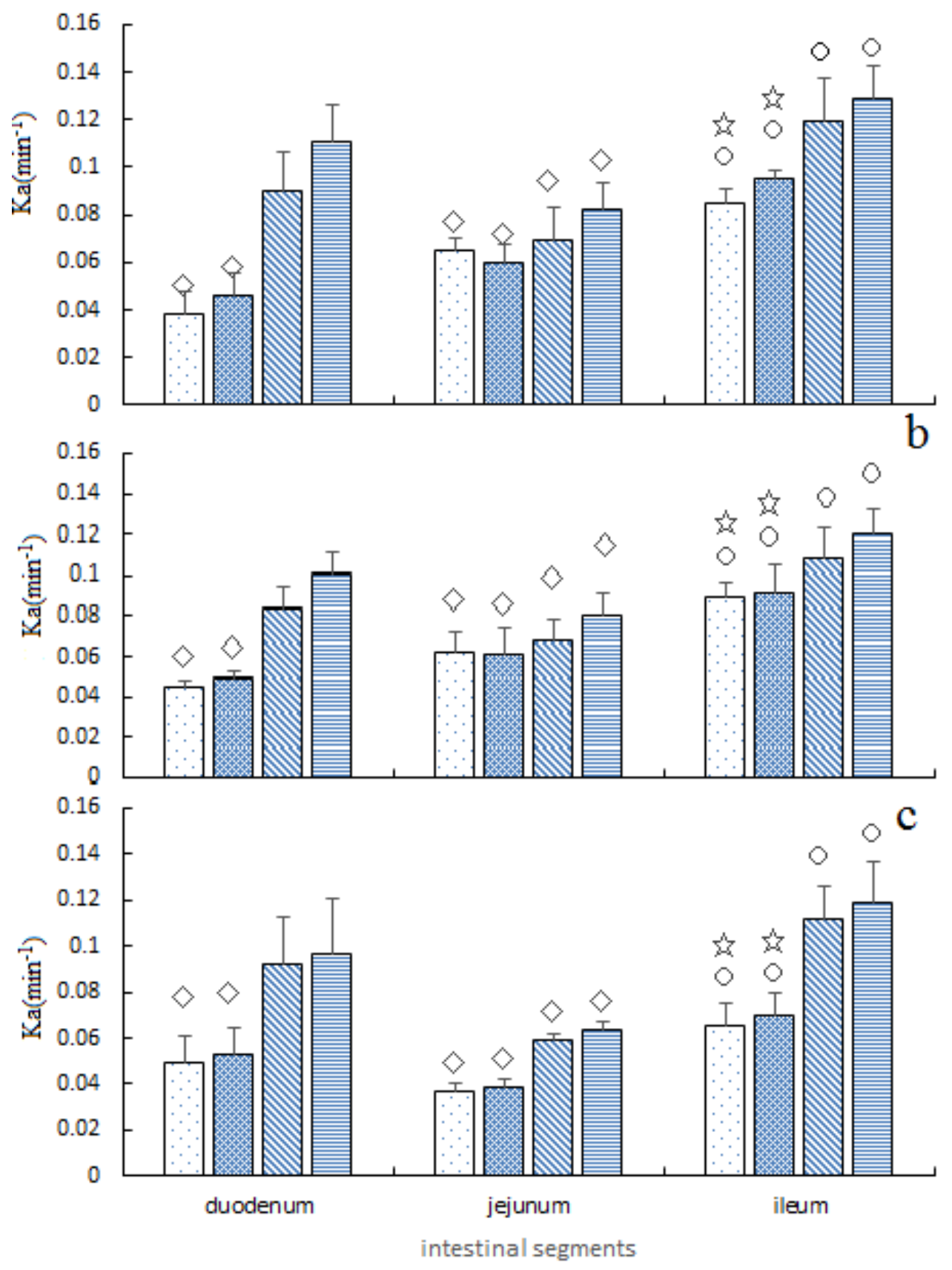

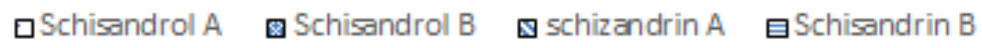

Figure 4: Absorption rate constant (Ka) of the four lignans at low (a), middle (b), high concentrations (c); is $p<$ 0.05 , VS. Ka of the duodenum; $\circ p<0.05$, VS. Ka of jejunum; $\diamond p<0.05$, VS. Ka of ileum 


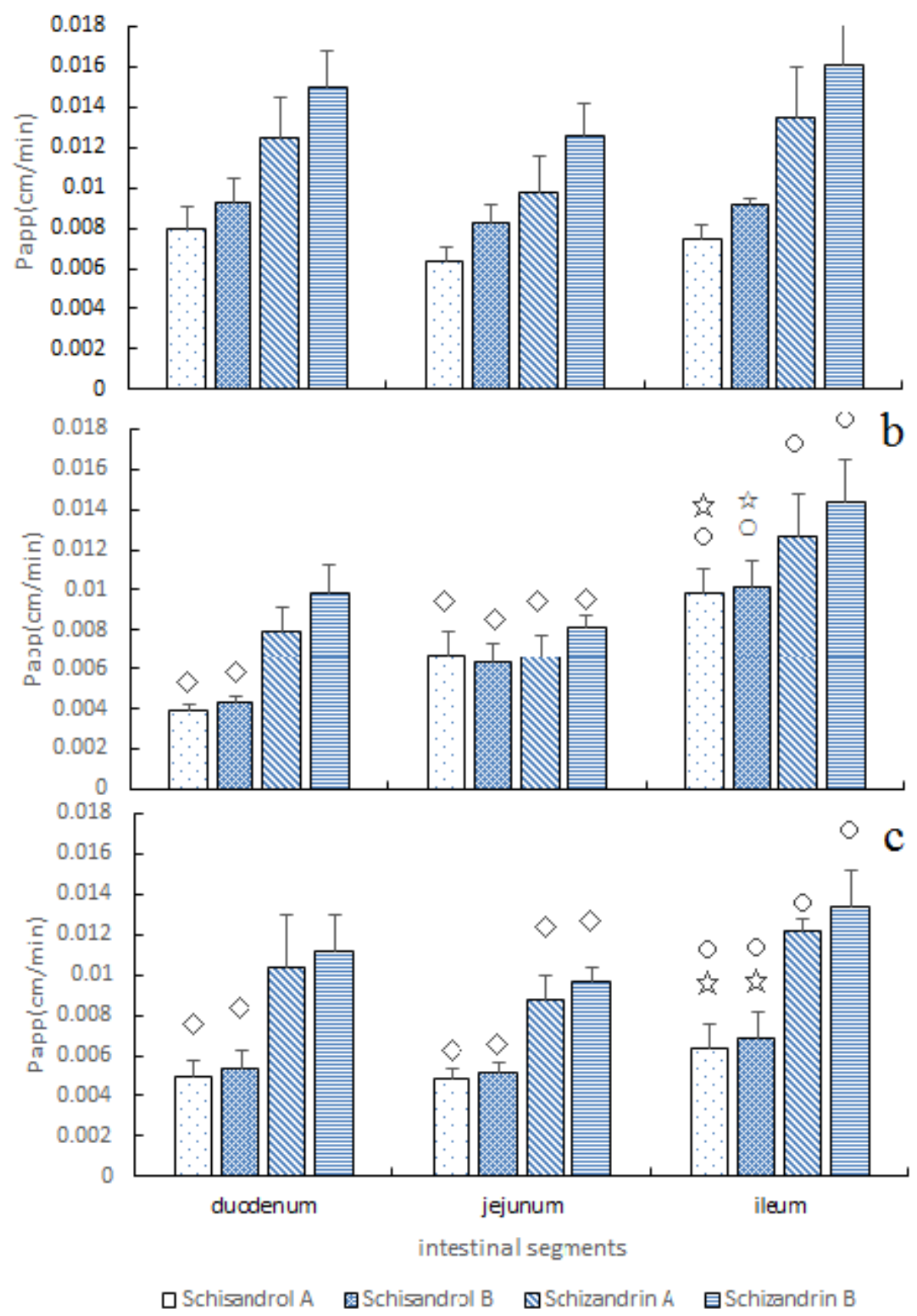

Figure 5: Apparent permeabilities (Papp) of the four lignans at the low (a), middle (b), high concentrations (c); is $p<0.05$, VS. Papp of the duodenum; $\circ p<0.05$, VS. Papp of jejunum; $\diamond p<0.05$, VS. Papp of ileum 


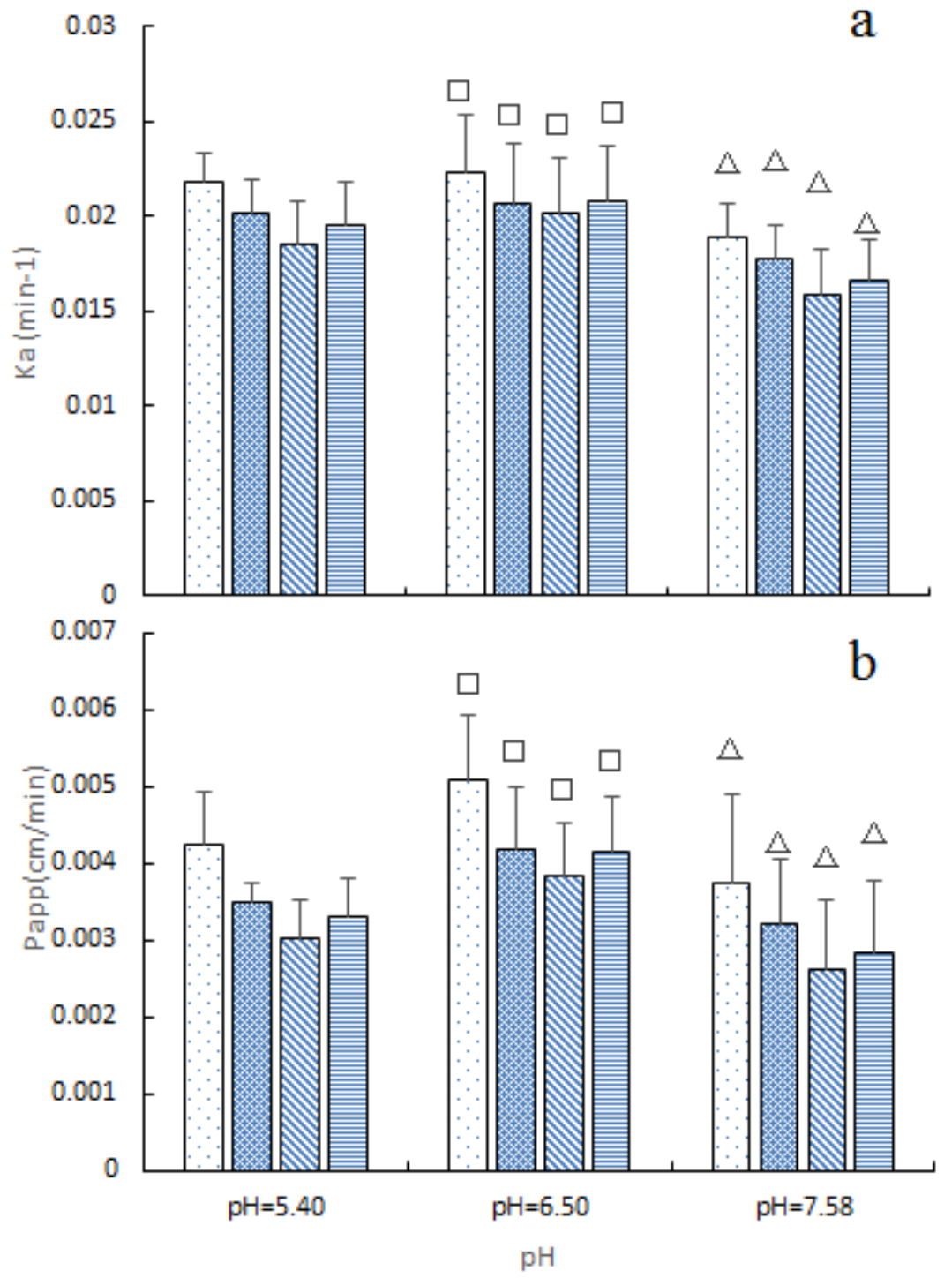

Figure 6. Ka (a) and Papp (b) of the four lignans at different $\mathrm{pH} ; \mathrm{s} p<0.05$, VS. the duodenum; $\circ p<0.05$, VS. the jejunum; $\diamond p<0.05$, VS. the ileum) 


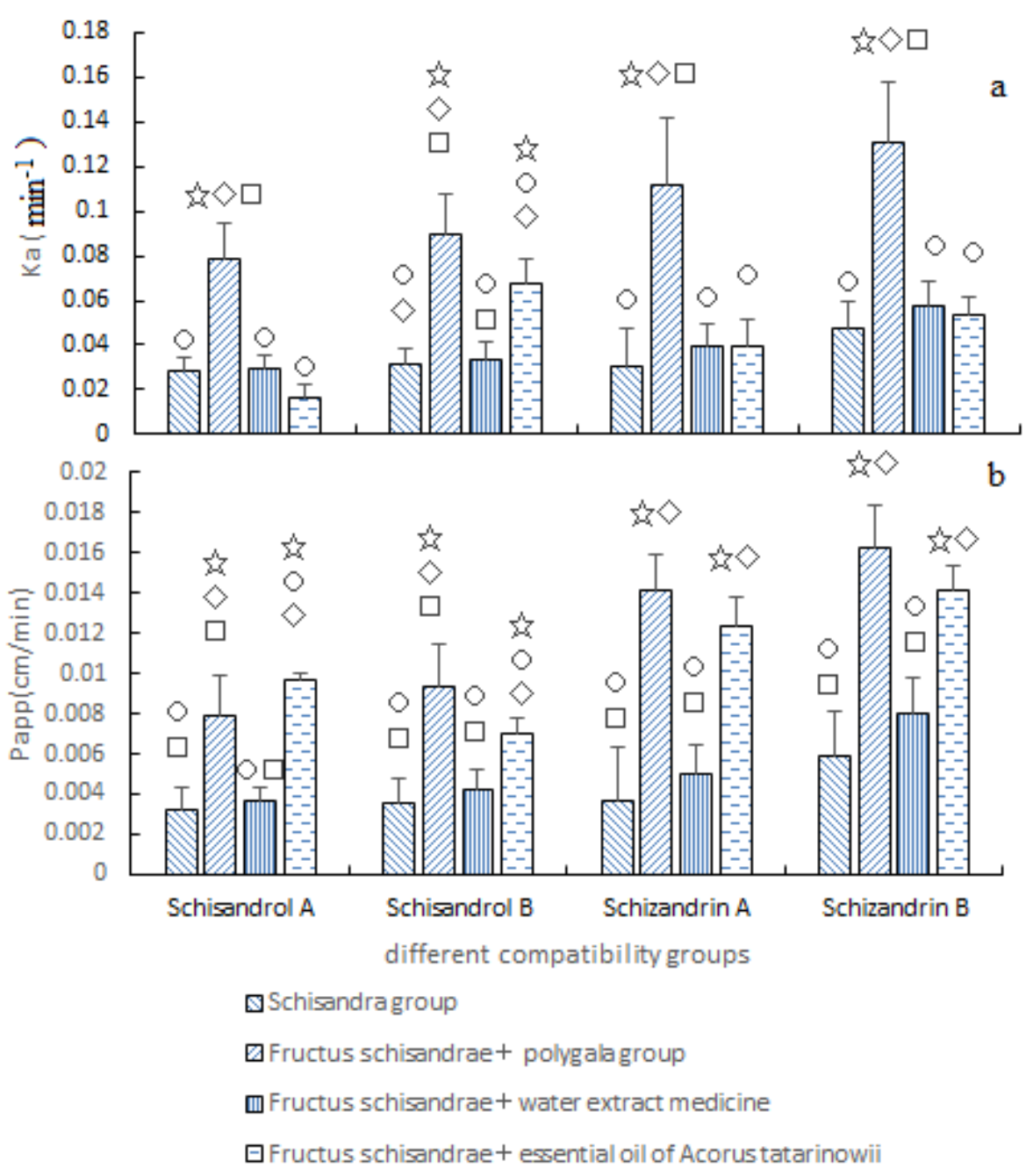

Figure 7: Ka (a) and Papp (b) of the four lignans in different compatibility groups; is $p<0.05$, VS. the Schisandra group; $\circ p<0.05$, VS. the Schisandra and polygala group; $\diamond p<0.05$, VS. the Schisandra and other water extraction group; $\square p<0.05$, VS. Schisandra and Acorustatatinowii Schott Naphtha group

\section{CONCLUSION}

The results in this study demonstrate that Polygala tenuifolia enhances the intestinal absorption of the four lignans via inhibition of the activity of intestinal P-gp, thereby improving their oral bioavailability. Comparison of the intestinal absorptions of the four lignans in Schisandra chinensis when perfused with a single herb and formula can help in understanding their compatibility mechanisms.

\section{DECLARATIONS}

\section{Acknowledgement}

This work was supported by the Ministry of National Science and Technique, China (no. 2014ZX09304306) and.Collaborative Innovation Construction Plan of Beijing University of Chinese Medicine (no. 2013-XTCX-03).

\section{Conflict of Interest}

No conflict of interest associated with this work.

\section{Contribution of Authors}

The authors declare that this work was done by the authors named in this article and all liabilities pertaining to claims relating to the content of this article will be borne by them.

\section{Open Access}

This is an Open Access article that uses a funding model which does not charge readers or their institutions for access and distributed under the terms of the Creative Commons Attribution License (http://creativecommons.org/licenses/by/ 4.0) and the Budapest Open Access Initiative (http://www.budapestopenaccessinitiative.org/rea d), which permit unrestricted use, distribution, and reproduction in any medium, provided the original work is properly credited. 


\section{REFERENCES}

1. Cheng N, Ren N, Gao H, Lei X, Zheng J, Cao W. Antioxidant and hepatoprotective effects of Schisandrachinensis pollen extract on $\mathrm{CCl} 4$-induced acute liver damage in mice. Food Chem Toxicol 2013; 55 (28): 234-240.

2. Cheng J, Zhang Z, Zheng Z, Lv G, Wang L, Tian B, Hua $Y$. Antioxidative and Hepatoprotective Activities of Deinoxanthin-Rich Extract from $D$. radiodurans $R 1$ against Carbon Tetrachloride-Induced Liver Injury in Mice. Trop J Pharm Res 2014; 13(4): 573-580.

3. Ekiert RJ, Szopa A, Ekiert H, Krzek J, Dzik E. Analysis of lignans in Schisandra chinensis fruits, leaves, biomasses from in vitro cultures and food supplements. $J$ Funct Foods 2013; 5(4): 1576-1581.

4. He XG, Lian LZ, Lin LZ. Separation and determination of active components in Schisandra chinensis Baill and its medicinal preparations by non-aqueous capillary electrophoresis. J. Chromatogr. A 1997; 757(1): 81-87.

5. Eun JJ, Hee KL, ki YL, Byung JJ, Dae HK; Jin HP, Joo $H S$, Jungmoo $H$, Jong $H L$, Sang HS. The effects of lignan-riched extract of Shisandra chinensis on amyloid$\beta$-induced cognitive impairment and neurotoxicity in the cortex and hippocampus of mouse. J Ethnopharmacol 2013; 146 (1): 347-354.

6. Zornoza T, Cano-Cebrian MJ, Nalda-Molina R, Guerri C, Granero L, Polache A. Assessment and modulation of acamprosate intestinal absorption: comparative studies using in situ, in vitro (CACO-2 cell monolayers) and in vivo models. Eur J Pharm Sci 2004; 22(1): 347-56.

7. Zhou Wei, Xiaobin Tan, Jinjun Shan, Ting Liu, Baochang Cai, Liuqing Di. Effect of chitooligosaccharide on the intestinal absorptions of phenylethanoid glycosides in Fructus Forsythiae extract. Phytomedicine 2014; 21(12): 1549-1558.

8. Kathleen M, Hillgren, Akira Kato, Ronald T, Borchardt. In vitro systems for studying intestinal drug absorption. Med Res Rev 1995; 15(2): 83-109.

9. Chan K. Chinese medicinal materials and their interface with Western medical concepts. J Ethnopharmacol 2005; 96(1): 1-18.

10. Jiang $C P$, He $X$, Yang $X L$, Zhang $S L$, Li H, Song ZJ, Zhang CF, Yang ZL, Li P. Intestinal absorptive transport of Genkwanin from Flos genkwa using a single-pass intestinal perfusion rat model. Am J Chin Med 2014; 42(2): 349-59.

11. Su S, Cui W, Duan JA., Hua Y, Guo J, Shang E, Liu P, Tang Y. UHPLC-MS simultaneous determination and pharmacokinetic study of three aromatic acids and one monoterpene in rat plasma after oral administration of Shaofu Zhuyu Decoction. Am J Chin Med 2013; 41(3): 697-715.
12. Liu HJ, Yu CP, Hsieh YW, Tsai SY, Hou YC. Inhibition of Monocarboxylate Transporter-Mediated Absorption of Valproic Acid by Gegen-Qinlian-Tang. Am J Chin Med 2013; 41(2): 369-378.

13. Holmstock N, Annaert P, Augustijns P. Boosting of HIV protease inhibitors by ritonavir in the intestine: The relative role of Cyp and P-gp inhibition based on Caco-2 monolayers versus in situ intestinal perfusion in mice. Drug Metab Dispos 2012; 40(8): 1473-1477.

14. Sun M, Zhai X, Xue K, Hu L, Yang X, Li G, Si L. Intestinal absorption and intestinal lymphatic transport of sirolimus from self-microemulsifying drug delivery systems assessed using the single-pass intestinal perfusion (SPIP) technique and a chylomicron flow blocking approach: linear correlation with oral bioavailabilities in rats. Eur J Pharm Sci 2011; 43(3): 132-140.

15. Zhou W, Di LQ, Wang J, Shan JJ, Liu SJ, Ju WZ, Cai BC. Intestinal absorption of forsythoside $A$ in in situ singlepass intestinal perfusion and in vitro Caco-2 cell models. Acta Pharmacol Sin 2012; 33(8): 1069-1079.

16. Escribano E, Sala XG, Salamanca J, Navarro CR, Regué $J Q$. Single-pass intestinal perfusion to establish the intestinal permeability of model drugs in mouse. Int $J$ Pharm 2012; 436(1): 472-477.

17. Valizadeh $H$, Mehtari $M$, Zakeri-Milani P. Evidence for enhanced intestinal absorption of digoxin by $P$ glycoprotein inhibitors. Trop J Pharm Res 2013; 11(6): 939-945.

18. Xue Han, Brian Y. Chow, Huihui Zhou, Nathan C. Klapoetke, Amy Chuong, Reza Rajimehr, Aimei Yang, Michael V. Baratta, Jonathan Winkle, Robert Desimone et al. A high-light sensitivity optical neural silencer: development and application to optogenetic control of non-human primate cortex. Front Syst Neurosci 2011; 5: 18.

19. Fan Yu, Chenghua He, Ayman Y. Waddad, Were L. L. Munyendo, Huixia Lv, Jianping Zhou, Qiang Zhang. Noctyl-N-arginine-chitosan (OACS) micelles for gambogic acid oral delivery. Drug Dev Ind Pharm2014; 40(6): 774782.

20. Zhen-Hai Zhang, Xiao-Pan Wang, Waddad Y. Ayman, Were L. L. Munyendo, Hui-Xia Lv, Jian-Ping Zhou. Studies on lactoferrin nanoparticles of gambogic acid for oral delivery. Drug Deliv 2013; 20 (2): 86-93.

21. Sutton SC, Rinaldi MTS, Vukovinsky KE. Comparison of the gravimetric, phenol red, and 14C-PEG-3350 methods to determine water absorption in the rat singlepass intestinal perfusion model. AAPS Pharm Sci 2001; 3(3): 1-5.

22. Sa $C, L v H, B a Y$, Sun J, Shi R. The effects of notoginsenoside $R 1$ on the intestinal absorption of geniposide by the everted rat gut sac model. Journal of Ethnopharmacology 2012; 142 (1): 136-143.

23. Fagerholm U, Johansson M, Lennernäs H. Comparison between permeability coefficients in rat and human jejunum. Pharm Res-Dordr 1996; 13(9): 1336-1342. 